\title{
Evaluating elbow joint kinematics with the Stewart Platform Mechanism
}

\author{
M. Alrashidi ${ }^{1}$, İ. Y1ldiz ${ }^{2}$, K. Alrashdan ${ }^{1} \&$ İ. Esat ${ }^{1}$ \\ ${ }^{1}$ School of Engineering and Design, Brunel University, UK \\ ${ }^{2}$ Mechanical Engineering Department, Yuldiz Technical University, \\ Turkey
}

\begin{abstract}
Joint measurement is necessary for studying joint laxity. Joint laxity in elbows is a problem which normally comes with age. However, it can increase to critical levels with rupture or damage to the ligaments of the elbow and affects the stability and capabilities of the joint, interfering even with daily activities.

A new Stewart platform based elbow joint measurement was developed. The study focused on flexion, extension, valgus and varus motions and all experimental data were taken from a real experimental setup. The centre of rotation and motion of the angles of the forearm were found by the kinematics of the Stewart Platform Mechanism with a simmechanics based program algorithm.

The joint motions of two male subjects were measured and the results of these measurements were compared with graphs. Comparisons demonstrate that the Stewart platform based measurement device sufficiently measures all motions of the elbow with six axes.
\end{abstract}

Keywords: joint laxity, Stewart platform, elbow kinematics.

\section{Introduction}

Kinematics of the elbow joint occupy a considerable place in orthopedic surgery. Many devices have been constructed with this aim. Hand goniometers were formerly employed for measuring elbow kinematics [1].

Morrey and Chao studied the motions of the elbow joint [2]. They measured elbow flexion and forearm rotation by using an electronic goniometer. Another study was published by Morrey and Chao for calculating elbow joint motion 
with the help of biplanar roentgenograms [3]. They obtained three-dimensional kinematics of the joint in their research.

Tanaka et al. used electromagnetic motion tracking data and described the first three-dimensional elbow kinematic [4].

Lateral roentgenograms used a kinematic analysis of elbow kinematics by London [5]. In this research, London used a special Reuleaux technique for analysis. The Reuleaux technique [11] was first used by Fisher to obtain he location of the axis of elbow flexion [6].

Bottlang et al. [7] used direct electromagnetic motion tracking to trace the passive and dynamic motion of the natural elbow joint.

With improving technology and silicone technology, inertial and magnetic sensors have also been employed for measuring human joints [8].

In this study, a Stewart platform based device was developed for measuring elbow kinematics. The Stewart platform mechanism was first proposed as a flight simulator in 1965 by Stewart [9].

\section{Materials and methods}

\subsection{Stewart Platform Mechanism}

The Stewart Platform Mechanism (SPM) is a six-axis parallel mechanism. This type of parallel mechanism is often applied in robotics and also in the medical field. A Stewart platform type robot has one moving plate, six parallel actuators and one fixed plate. Actuators are usually mounted between the moving plate and the fixed plate by spherical or universal joints. This configuration allows the mechanism to have three translational and three rotational motions.

In this study a Stewart platform type measurement device was used. This device also has one moving plate and one fixed plate, but instead of actuators it has six linear potentiometers (Fig. 1).

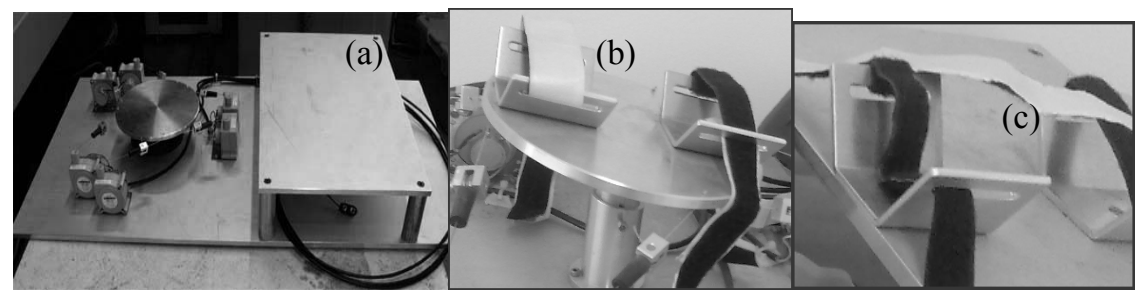

Figure 1: $\quad$ Stewart platform based elbow joint measurement device (a), arm fixation apparatus $(b, c)$.

\subsection{Measurement method}

The SPM is a kind of kinematic chain. In this study, potentiometers were used as the legs of the SPM. Potentiometer data were used for calculating the elbow kinematics. An illustrative block diagram to illustrate the method of measurement method is found in Fig. 2. 


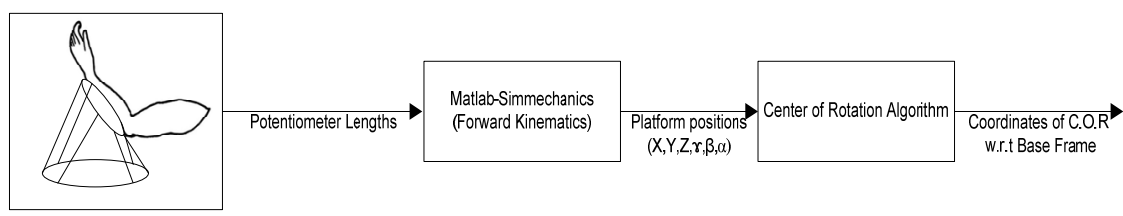

Figure 2: $\quad$ Measurement steps.

As shown in Fig. 2, the first step is to fix the subject's forearm to the SPM. The next step involves gathering the potentiometer data from the data acquisition device while the forearm is moving. Calculating the position of the centre of gravity of the SPM using the model of SPM derived from Matlab Simmechanics constitutes the third step in measuring. The Matlab Simmechanics model includes the forward dynamics and kinematics of the SPM. The forward kinematics method is one of the critical parts of the measuring. In parallel mechanisms such as the SPM, it is extremely difficult to derive the positions of the centre of gravity from the leg lengths. Many methods have been developed for solving this problem. The most important one is the Newton-Rhapson method, which entails an iterative solution [10]. The block diagram of a virtual Simmechanics model of SPM is shown in Fig. 3. The final step allows us to obtain the elbow kinematics from the position of the SPM.

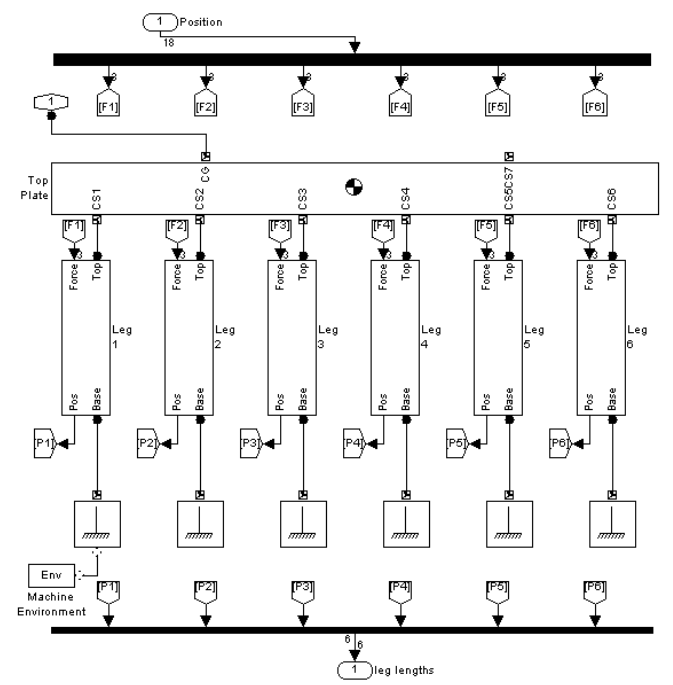

Figure 3: Matlab-Simmechanics model of the SPM. 


\subsection{Elbow kinematics}

The SPM has three translational $(x, y, z)$ and three rotational $(\gamma, \beta, \alpha)$ motions with respect to its base frame, as shown in Fig. 5a. Rotational motions $\gamma, \beta, \alpha$ represent the rotations of the mobile platform along the $x, y, z$ axes, respectively.

Four main motions of the forearm (flexion, extension, varus and valgus) were observed and measured on the subjects. The relationship between these motions and the SPM motions are shown in Fig. 4.
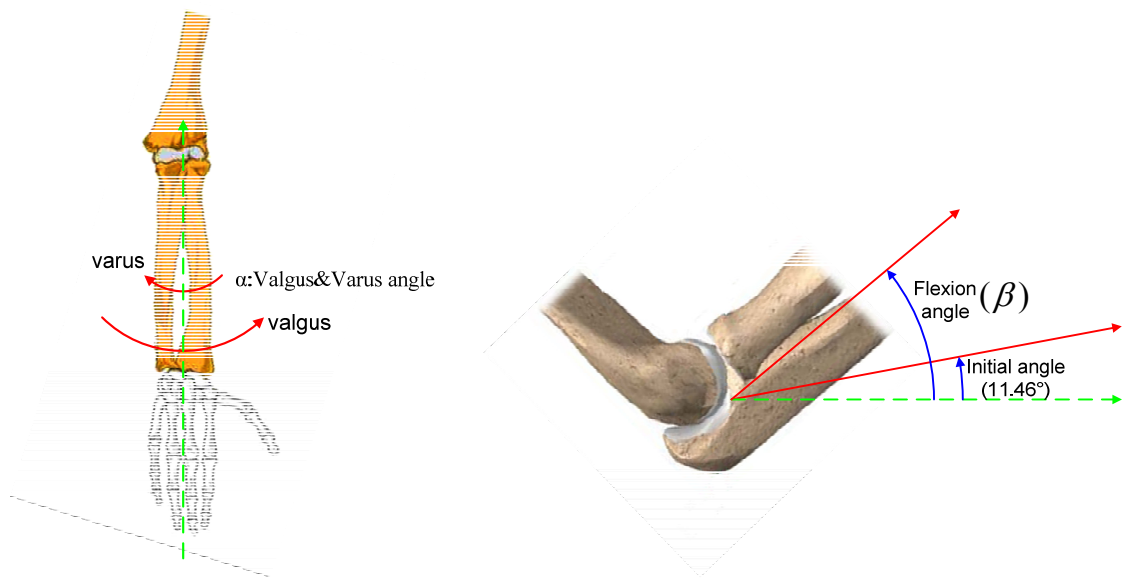

Figure 4: Angle definitions of forearm.

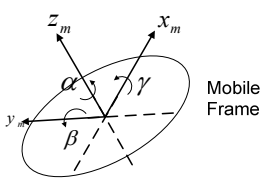

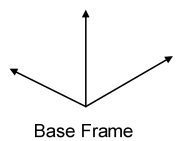

a

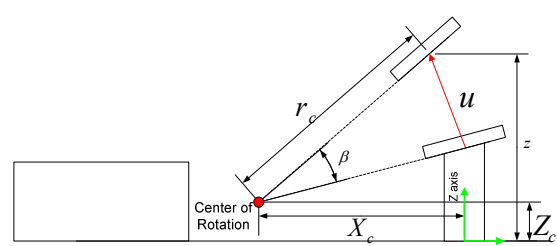

b

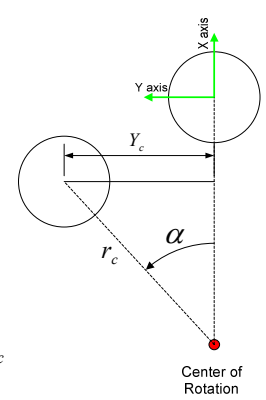

C

Figure 5: (a) Base and mobile frames; (b) side view of setup; and (c) top view of mobile platform.

Obtaining the coordinates of the rotation centre is significant when treating diseases of the forearm. Almost all elbow implants have one rotation centre 
because they are fully mechanical structures and not biological structures. The normal elbow is a flexible joint thanks to its ligaments and this means that it has no exact rotational centre. The laxity of the elbow joint can be expressed in the space of the centre of rotation. The advantage of the Stewart measurement device is that it makes all motions of arm measurable not only evident motions such as flexion and extension, but also negligible motions, including all six of its axes.

In both the side and the top view of SPM, it is clearly shown (see Fig. 5b-c) that the forearm draws an imaginary circle which has the radius $\left(r_{c}\right)$. According to the cosine theorem,

$$
u^{2}=r_{c}^{2}+r_{c}^{2}-2 r_{c} r_{c} \cos \beta
$$

$u$ indicates the distance between the initial point and current point of the centre of gravity of the mobile platform and $\beta$ is the angle of flexion. The rotation radius can be obtained by extracting $r_{c}$ in Equation 1 .

$$
r_{c}=\sqrt{\frac{u^{2}}{2-2 \cos \beta}}
$$

Finally, the coordinates of centre of rotation of the elbow joint can be expressed as:

$$
\begin{aligned}
& X_{c}=-r_{c} \cdot \cos \beta \\
& Y_{c}=r_{c} \cdot \sin \alpha \\
& Z_{c}=Z-r_{c} \cdot \sin \beta
\end{aligned}
$$
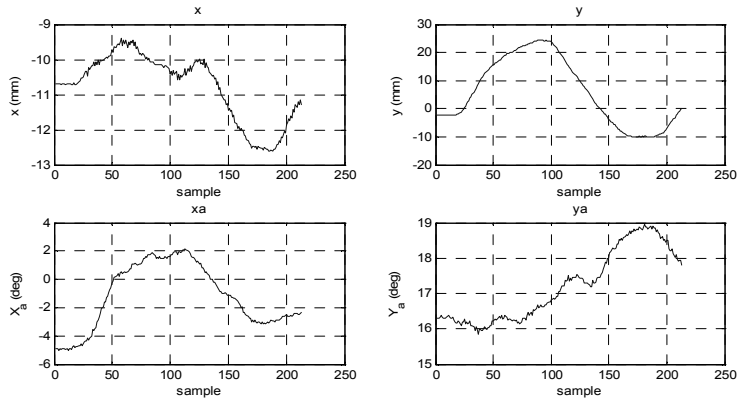

ya
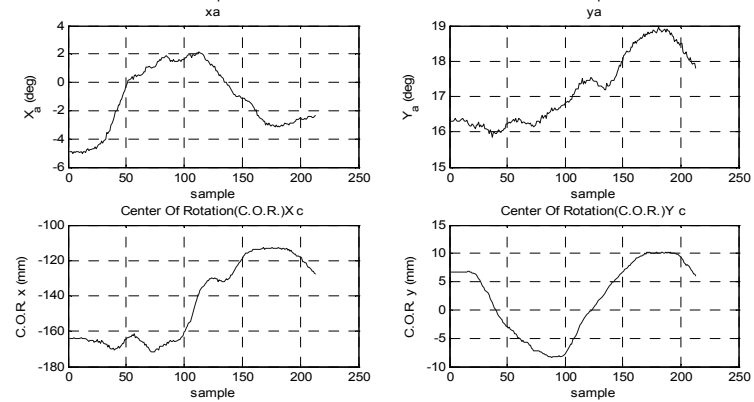
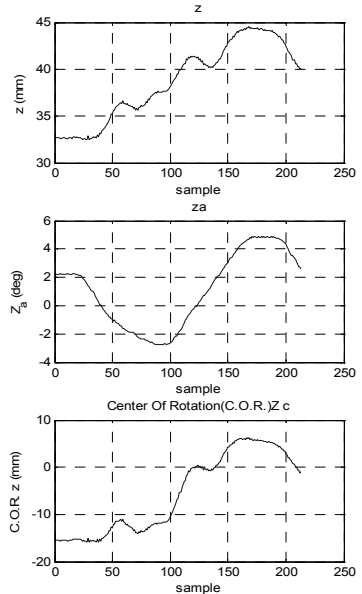

Figure 6: Motions of SPM and centre of rotation during the vargus and valus motions. 


\section{Results}

The forearms of two subjects were tested by the SPM based measurement mechanism. The motions of the SPM and centre of rotation can be seen in Fig. 6 .

In Fig. 6, the $x, y, z$ graphs demonstrate the translational motion of the SPM. $X_{a}, Y_{a}, Z_{a}$ depict the angular motions of the SPM, which are $\gamma, \beta, \alpha$. The last three graphs illustrate the centre of rotation of the forearm. All the graphs in Fig. 6 were created from the data taken from one subject during the vargus and valus motions and were not created with the aim of comparison. They show that the SPM-based measuring mechanism allows all six axes to be measured.

Flexion and extension motions are shown in Fig. 7. The flexion angles are limited to about 80 degrees because the lengths of potentiometers are themselves limited.
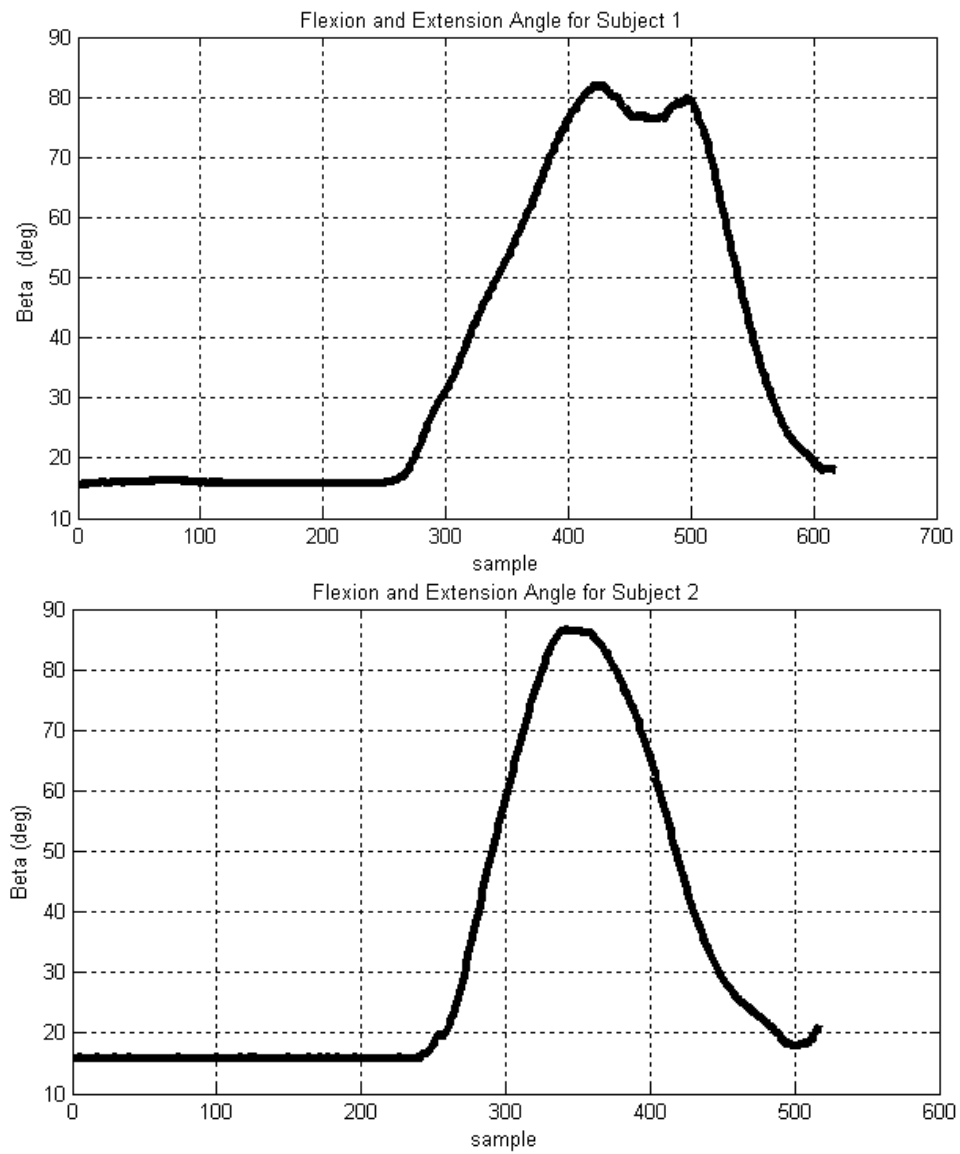

Figure 7: Flexion and extension angles. 
The varus and valgus motions are shown in Fig. 8. In the first graph, the subject starts with varus motion and follows it with valgus motion. Both graphs illustrate that the varus and valgus motion capacities are limited to a minimum of 5 degrees.
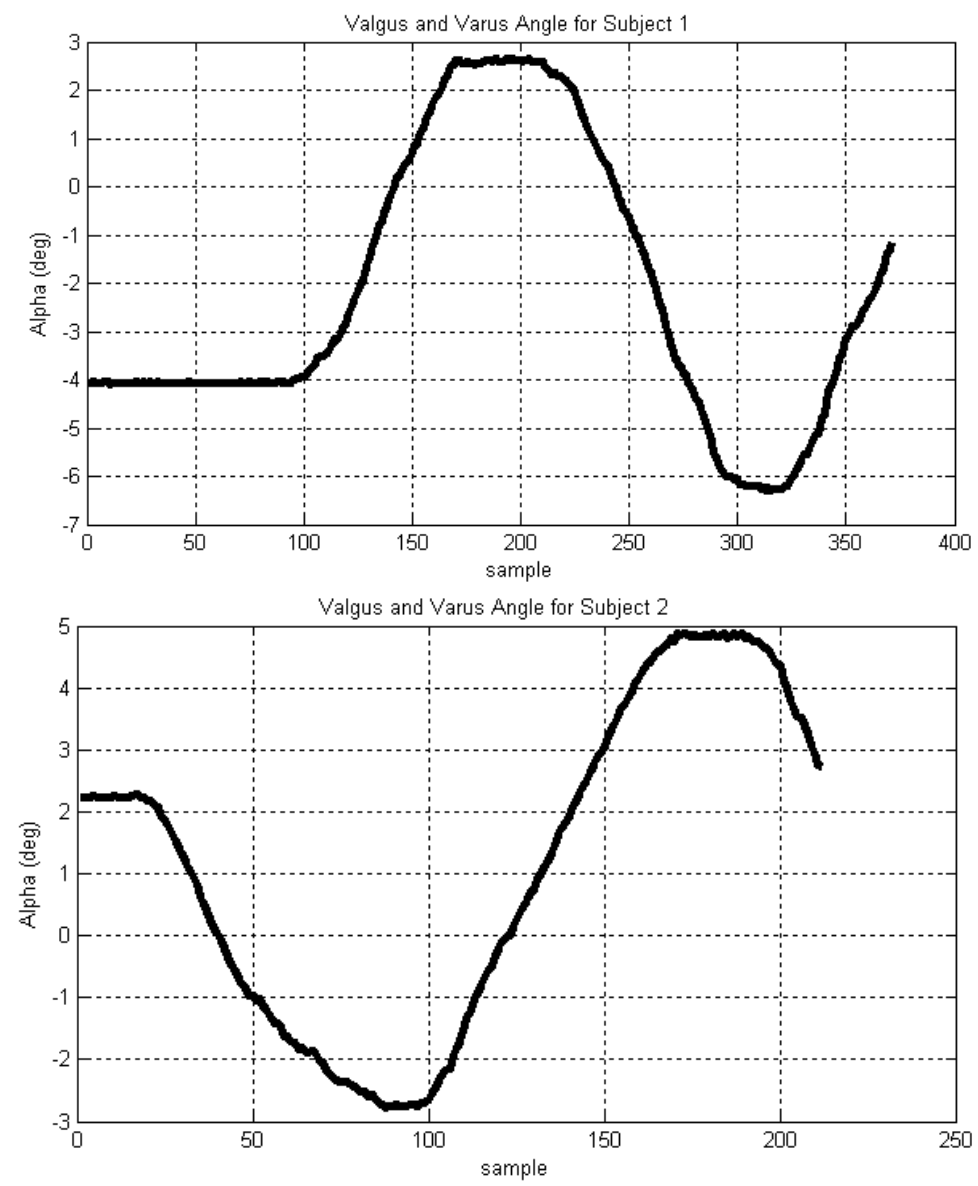

Figure 8: Valgus and varus angles.

Fig. 9 illustrates the centre of rotation of the forearm on the $\mathrm{YZ}$ axis during valgus and valus motions, exposing joint laxity. Joint laxity is directly related to the bounds of the links so this is the main reason for selecting both valgus and varus motion. Values are a little bigger than expected, because fixing both forearm and shoulder to the set up is insufficiently exact.

\section{Conclusion}

A Steward platform based elbow joint measurement device is tested with basic motions of the forearm. The mechanism was tested with two subjects and 

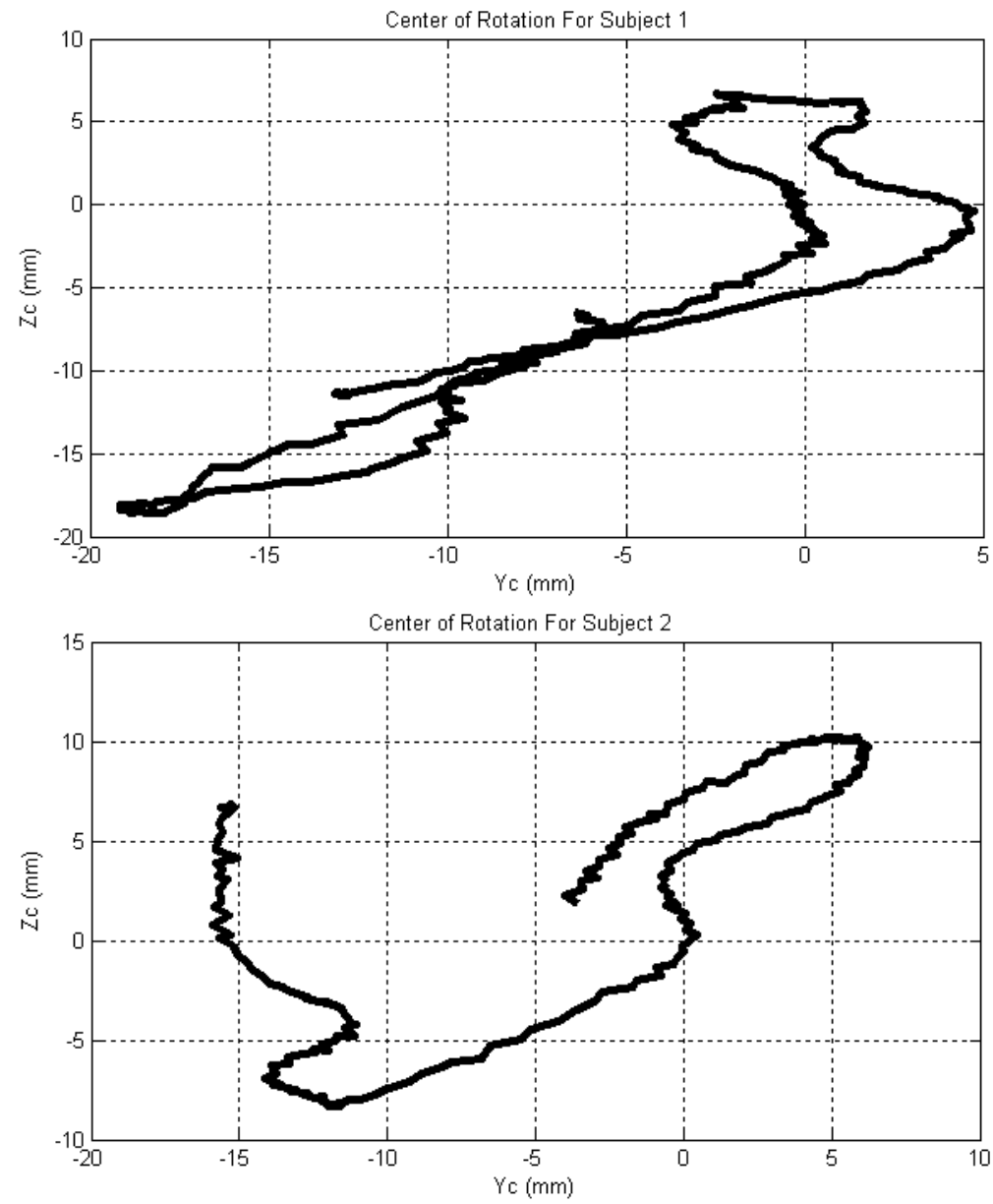

Figure 9: Joint laxity: valgus and varus.

succeeded in measuring all the motions of the forearm and ascertaining the centre of rotation. Tests were executed with the help of another person to ensure that the subjects made the specified motions.

The accuracy of the device was found insufficient because of the way in which the arm was fixed. Using special clamps should make the device more accurate.

In further works, a new SPM based joint measurement device with forced feedback should more accurate and should gather more comparable data with the help of forced feedback and linear actuators.

\section{References}

[1] Boone D.C. and Azen S.P.: Normal Range of Motion of Joints in Male Subjects. J. Bone and Joint Surg., 61-A:756-759, July 1979 
[2] Morrey B.F., Askew L.J. and Chao E.Y.: A Biomechanical Study of Normal Functional Elbow Motion. J. Bone and Joint Surg., 63-A:872-877, July 1981

[3] Morrey B.F. and Chao E.Y.: Passive Motion of the Elbow Joint. J. Bone and Joint Surg., 58:501-508, 1976

[4] Tanaka S., An K.N., Morrey B.F.: Kinematics and Laxity of Ulnohumeral Joint under Varus-Valgus Stress. J. Musculoskel Res 2:45-54, 1998

[5] London J.T.: Kinematics of the Elbow. J Bone and Joint Surg., 63:529535,1981

[6] Fisher G.: cited in R. Fick: Handbuch der Anatomie und Mechanik der Gelenke unter Berücksichtigung der Bewegenden Muskeln, Vol.2. p. 299, 1911

[7] Bottlang M., Madey S.M., Steyers C.M., Marsh J.L., Brown T.D.: Assessment of Elbow Joint Kinematics in Passive Motion by Electromagnetic Motion Tracking. J. Orthop Res, Vol.18:195-202,2000

[8] Cutti A.G., Giovanardi A., Rocchi L., Davalli A., Sacchetti R.: Ambulatory Measurement of Shoulder and Elbow Kinematics through Inertial and Magnetic Sensors. Med Bio Eng Comput. 46:169-178,2008

[9] Stewart, D., A Platform with Six Degrees-of-freedom, Proceedings of Mechanical Engineering Part I, Vol.180, pp. 371-386, 1965-1966.

[10] Harib K. and Srinivasan K.: Kinematic and Dynamic Analysis of Stewart Platform Based Machine Tool Structures. Robotica Vol:21,pp.541554.2003

[11] Reuleaux, Franz: Kinematics of Machinery: Outlines of Theory of Machines. pp. 56-80.Translated and edited by A.B. Kennedy. New York, Dover.1963 